\title{
Myogenic stem cells for the therapy of primary myopathies: wishful thinking or therapeutic perspective?
}

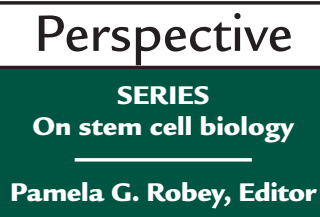

\author{
Giulio Cossu $^{1,3}$ and Fulvio Mavilio ${ }^{2}$ \\ ${ }^{1}$ Stem Cell Research Institute and \\ ${ }^{2}$ Gene Therapy Program, Istituto Scientifico H. San Raffaele, Milano, Italy \\ ${ }^{3}$ Department of Histology and Medical Embryology, University of Rome "La Sapienza," Rome, Italy
}

Address correspondence to: Giulio Cossu, Department of Histology and Medical Embryology, University of Rome "La Sapienza," Via A. Scarpa 14, 00161 Rome, Italy. Phone: 3906-4976-6757; Fax: 3906-446-2854;

E-mail: cossu@uniroma1.it.

Primary myopathies: the long road from gene identification to therapy

Primary myopathies are characterized by a progressive wasting of skeletal muscle that leads to deterioration of movements and, in the most severe cases, such as in Duchenne's muscular dystrophy (DMD), to complete paralysis and death. Most myopathies in which the molecular defect has been identified are due to mutations affecting proteins that form a supramolecular link between the cytoskeleton and the extracellular matrix, such as dystrophin, the mutated protein in DMD (1). In the absence of one of these proteins, mechanical stress associated with contraction progressively leads to degeneration of the muscle fiber, although the underlying mechanisms are still poorly understood. In the first phase of the disease, new muscle fibers are formed by fusion of resident myoblasts, called satellite cells (2), which also bear the molecular defect of the fibers that they replace, and hence undergo the same fate. Once the proliferation potential of satellite cells is exhausted, there is no further regeneration and the skeletal muscle is replaced by connective tissue.

The current therapeutic approaches to DMD involve pharmacological suppression of the inflammatory and immune responses, which usually provides only modest and temporary beneficial effects. Future approaches depend on cell and gene therapy technology and will require different strategies, none of which are currently ready to enter clinical practice. These range from the design of efficient, nonantigenic gene transfer vectors for in vivo gene therapy (3), to pharmacological upregulation of the synthesis of utrophin, a related protein that compensates for the loss of dystrophin (4), to myoblast transplantation, the focus of this Perspective. We will briefly review ten years of hopes and failures, in an attempt to predict whether, and when, transplantation of myogenic or pluripotent stem/progenitor cells might lead to significant clinical benefits for patients affected by DMD or other severe forms of muscular dystrophy.

Transplantation of heterologous myoblasts: from failure to new hopes

In 1989, Partridge and his collaborators showed that intramuscular injection of $\mathrm{C} 2 \mathrm{C} 12$ cells, an immortal myogenic cell line derived from adult satellite cells, could efficiently reconstitute dystrophin-positive, apparently normal fibers in dystrophic $m d x$ mice (5). This finding inspired a number of problematic attempts in the early 1990s to apply this strategy clinically, but using non-immortal myoblasts in patients. Myogenic cells isolated from immune-compatible donors were expanded in vitro and injected into specific muscles of DMD patients. All trials failed, for a number of reasons, some of which could have been predicted. C2C12 cells have unlimited life-spans and are syngeneic with $m d x$ mice, features that are lacking in normal human donor cells. Other difficulties only became apparent long afterwards. We now know that most myoblasts (up to 99\%) succumb soon after injection, due first to an inflammatory and then to a cellmediated immune response (6). The latter is directed against not only donor cell antigens but also against the wild-type dystrophin protein (7), which presents as a new antigen for both $m d x$ mice and DMD patients. We also know that the cells that survive this initial catastrophe do not migrate more than a few millimeters away from the injection site, indicating that countless injections would be required to provide a significant distribution of donor cells into the patient's muscles.

Through the years, the work of several laboratories has focused on these problems and has produced a stepwise, progressive increase in the recovery, survival, and colonization efficiency of injected myoblasts in mouse models. Satellite cells, previously defined on an anatomical basis, were found to comprise a hierarchy of muscle precursors, defined by their growth kinetics in vitro and their efficiency in repopulating muscle in vivo $(8,9)$. Partial immune suppression, injection of neutralizing antibodies directed against surface molecules of infiltrating cells (e.g., lymphocyte function-associated antigen 1 [LFA-1]), pretreatment of myoblasts in vitro with growth factors, and modification of the muscle connective tissue all contributed to improved myoblast survival upon injection in vivo (10). Recent extension of these types of protocols into primates (11) raises the possibility that better designed clinical trials may be on the horizon, with much more preclinical information in hand and clearer definitions of clinically relevant endpoints. 
Ex vivo therapy with autologous, engineered myoblasts

The ex vivo approach to gene therapy of primary myopathies was designed to overcome at least some of the immunological problems linked to myoblast transplantation. In this strategy, cells are isolated from a muscle biopsy, expanded in vitro, transduced with an appropriate vector encoding the therapeutic gene, and finally reinjected into one or more muscles of the patient from which they had been initially isolated (12). This approach, however, faces two additional problems: first, the difficulty of producing an appropriate, integrating vector to accommodate the very large dystrophin or utrophin cDNAs; and second, the limited life-span of myogenic cells isolated from dystrophic patients. The proliferative potential of human myogenic precursors declines considerably during early postnatal growth, in parallel with the progressive reduction in telomere length which occurs in the first 2 decades of life (13). Given the extra number of cell divisions that myogenic cells from DMD patients undergo in vivo during the various cycles of fiber degeneration and regeneration, their replication capacity is dramatically decreased during early childhood and continues to drop during the first decade of life. These cells are recovered in low number from muscle biopsies, grow poorly in vitro, and rapidly undergo senescence, even though they can be transduced by retroviral vectors with an efficiency comparable to that of normal cells $(14,15)$. This makes it very difficult to obtain reasonable numbers of genetically modified cells ex vivo and consequently lowers the expectations that this type of strategy might become practical.

Attempts to solve the problem of the limited life-span have relied on either immortalization of myogenic cells or recruitment of nonmyogenic cells with a higher proliferative potential. Immortalization of mammalian cells by oncogenes has been successful for decades, and in the case of molecules such as the large T antigen of SV40 or polyoma virus, immortalization is still compatible with a certain degree of differentiation into mature muscle fibers $(16,17)$. Nevertheless, safety concerns related to the persistence of an active oncogene in the genome of the immortalized cells have precluded, so far, any clinical application of this strategy. In order to overcome these concerns, we designed a retroviral vector expressing the wild-type simian vacuolating virus 40 (SV40) large $T$ antigen and harboring two lox sites in the long terminal repeats. Human primary myogenic cells transduced with this vector showed extended life-spans and retained their differentiation capacity. Transient expression of Cre recombinase allowed the entire provirus to be excised in $>90 \%$ of transduced cells, which then underwent terminal differentiation in vivo with an efficiency comparable to that of untreated, primary myogenic cells (18). Reversible immortalization thus allows primary myoblasts to be expanded in culture without compromising their ability to differentiate in vivo and could represent a safe means to increase the availability of these cells for clinical application. Alternative strategies might be conceived along similar lines, such as expressing active telomerase or inhibiting the expression of anti-oncogenes.

To increase the availability of primary myoblasts, we and others have also explored the possibility of using the $M y o D$ gene to induce myogenic conversion of nonmuscle, autologous cells such as skin fibroblasts, the growth potential of which is uncompromised in all primary myopathies. Fibroblasts can be obtained from a number of accessible sources (e.g., skin), easily expanded, and genetically modified in culture. If transduced at high efficiency with $M y o D$, for instance by transient exposure to an adenoviral vector, cultured fibroblasts irreversibly withdraw from the cell cycle and undergo myogenic differentiation both in vitro and in vivo $(19,20)$. These data indicate an alternative approach to gene therapy of primary myopathies, based on implantation of large numbers of genetically modified primary fibroblasts directed toward myogenesis by transient expression of MyoD ex vivo.

Many of the technical hurdles limiting the feasibility of myoblast transplantation have therefore been addressed with promising complementary approaches, which may provide the basis for a new round of clinical trials in the near future. More recently, however, unexpected findings have modified our thinking about myogenesis and myogenic cells and unveiled new therapeutic perspectives.

\section{A circulating myogenic progenitor from the bone marrow}

Spontaneous activation of a myogenic differentiation program in cells of mesodermal origin cocultured with muscle cells or injected into regenerating muscle in vivo has been known for many years (reviewed in ref. 21). Myogenic conversion, as this phenomenon is called, is rare and inefficient under normal conditions, although it has been observed in cells derived from tissues as diverse as the central nervous system, dermis, and bone marrow. The use of a transgenic mouse line in which a lac $Z$ gene is expressed under the control of muscle-specific regulatory elements (MLC3F-nlacZ) proved to be critical for these studies. In these animals, the $l a c Z$ reporter is expressed only in the nuclei of striated muscle (22) and serves as a faithful and sensitive marker of myogenic differentiation, both in vitro and in vivo. When nonmyogenic cells from MLC3F-nlac Z mice are injected into regenerating muscle of immunodeficient mice, myogenic conversion of donor cells is scored by the formation of muscle fibers with centrally located, $\beta$-gal-positive nuclei. By this type of assay, we observed that unfractionated bone marrow, even when depleted of fibroblasts and other adherent cell components, gives rise to labeled muscle fibers with an unexpectedly high efficiency. Rather than myogenic conversion, these experiments suggested the existence of progenitor cells endowed with myogenic potential within the bone marrow.

Bone marrow is a specialized environment for development, maintenance, and differentiation of pluripotent cells, which include the hematopoietic stem cell and the stroma-derived stromal stem cell, a long-lasting precursor of bone, cartilage, and other connective 
tissues in mice and in humans (23; see also Bianco and Robey, this Perspective series, ref. 24). Although stroma-derived cells were reported to give rise to sporadic myotubes in culture upon induction with 5-azacytidine (23), these cells do not differentiate into muscle under normal conditions, either in vitro or in vivo, suggesting that the bone marrow-derived myogenic progenitor may be a different cell entity (25).

Since direct injection into the muscle is an inefficient and impractical route of cell delivery, bone marrow-derived progenitors immediately presented themselves as an alternative to myoblasts and satellite cells, provided that they could be systemically rather than locally delivered to a dystrophic muscle. To test this possibility, we transplanted MLC3F-nlacZ bone marrow into lethally irradiated scid/bg mice, allowed the transplanted bone marrow fully to reconstitute the recipient animals, and then induced muscle regeneration by standard cardiotoxin injection into a leg muscle. Histochemical analysis unequivocally showed the presence of $\beta$-gal-positive nuclei at the center and periphery of regenerated fibers. These results demonstrated, for the first time to our knowledge, that murine bone marrow should contain transplantable progenitors that can be recruited to an injured muscle through the peripheral circulation, and that participate in muscle repair by undergoing terminal differentiation into mature muscle fibers (26). Publication of this report in 1998 raised new interest in myogenic progenitors and much speculation as to their possible clinical use. If confirmed in humans, the existence of a transplantable cell that could be systemically delivered to a large fraction of muscles would open a new avenue in the development of a cellmediated replacement therapy for muscular dystrophy.

However, in spite of these promising results, the real therapeutic potential of such a strategy remained questionable. Quantitatively, muscle repair by bone marrow-derived cells appeared in fact as a marginal phenomenon. Less than $0.5 \%$ of the regenerated fibers contained $\beta$-gal-positive nuclei in the muscles of the transplanted mice. On the other hand, a number of factors inherent to the experimental model obviously limit the overall efficiency of the system. First, bone marrow was transplanted in toto and according to a protocol optimized for hematopoietic stem cells. Under these conditions, not only is the relative abundance of myogenic progenitors unknown, but, given the absence of any selective advantage, even their transplantation efficiency is difficult to determine. Second, resident myogenic precursors are perfectly healthy in scid/bg mice and are unaffected by the low-dose radiation administered before transplantation. Endogenous cells may therefore compete effectively with marrow-derived cells for muscle repair. In fact, our experiments indicate that marrow-derived progenitors have a slower differentiation kinetics compared with satellite cells, which respond faster to the regeneration stimulus and probably carry out much of the repair by the time marrow-derived cells come into the picture. This kinetic difference might suggest that marrow-derived progenitors represent a more primordial precursor than a satellite cell. This hypothesis is difficult to demonstrate because the
MLC3F-nlacZ transgene is activated only in cells actively undergoing myogenic differentiation and differentiated fibers, but not in quiescent myoblasts (22). This overall picture might change substantially in a dystrophic background, where a pool of normal or genetically corrected cells could enjoy a selective advantage and effectively replace a progressively depleted pool of defective muscle cells. The real potential of bone marrow transplantation as a muscle replacement therapy therefore needed further testing in such a model.

The result of this type of testing came in mid-1999 from experiments carried out by Gussoni et al. Dystrophin-deficient $m d x$ mice, when transplanted with the bone marrow of syngeneic C57BL/10 mice, developed a small number of dystrophin-positive fibers containing genetically marked ( $\mathrm{Y}$ chromosome) donor nuclei, 8-12 weeks after transplantation (27). Although the efficiency of muscle repopulation by marrow-derived progenitors appeared to be slightly higher in this model, the number of fibers carrying both dystrophin and the Y chromosome never exceeded $1 \%$ of the total fibers in the average muscle. Thus, even in a chronically regenerating environment, marrow-derived progenitors are unable to give rise to new muscle fibers in clinically relevant numbers. Similar experiments carried out in a slightly different animal model, the $m d x 4 c v$ mutant (which exhibits an extremely low background of revertant fibers), showed that the number of donor-derived, dystrophin-positive fibers does not increase with time and never exceeds $0.5 \%$ of the total fibers as long as 1 year after bone marrow transplantation (our unpublished observations).

From the clinical point of view, the studies on the $m d x$ mice were a disappointment. They clearly indicated either that the number of myogenic progenitors present in a bone marrow transplant is insufficient to produce enough muscle mass, or that they are not transplanted as efficiently as the hematopoietic progenitors by standard bone marrow transplantation procedures. It is also possible that the $m d x$ background does not provide enough selective advantage to these cells to trigger their expansion and ultimately a significant replacement of the resident satellite cell pool. In fact, one might argue that, despite the dystrophin defect, the $m d x$ mouse does not develop a dystrophic clinical phenotype and that its musculature is actually hypertrophic. Hence, $m d x$ satellite cells may be perfectly capable of regenerating muscle for most of the animal's life-span. The clinical picture is dramatically different in human muscular dystrophy, where the repair potential of muscle satellite cells is lost in the first years of a patient's life and skeletal muscles undergo progressive and irreversible degeneration. In this situation, dystrophin-positive fibers made by transplanted bone marrow progenitors might have a selective advantage, resist degeneration, and progressively replace dystrophin-negative fibers. Therefore it is difficult to extrapolate the results obtained in $m d x$ mice to humans, and a different animal model is clearly needed to address any efficiency issue in a preclinical setting. 
Pluripotent stem cells: biological curiosity or new hope for cell transplantation?

In the course of their bone marrow transplantation studies, Gussoni et al. made the important observation that the muscle of C57BL/10 mice contains a population of primitive cells (side population [SP] cells) that share the property of excluding a particular fluorescent dye (HoEchst 33342) with a subfraction of bone marrow-derived primitive hematopoietic stem cells (28). Like bone marrow SP cells, muscle SP cells are capable of long-term hematopoietic reconstitution upon transplantation into $m d x$ mice, and both marrow and muscle SP cells give rise to dystrophin-positive muscle fibers 8-12 weeks after transplantation (27). At the same time, but by using a different technique which includes tissue culture steps, Jackson et al. showed that muscle tissue is a source of cells that are capable of long-term hematopoietic reconstitution in a serial transplantation assay and are even more abundant in muscle than in the bone marrow itself (29). Together with another, unrelated report on the multipotential nature of neural stem cells (30), these data provided rather unexpected evidence that hematopoietic stem cells may reside outside the bone marrow and suggested the existence of pluripotent cells capable of differentiation into both the hematopoietic and the muscle cell lineage. The term "plasticity," used for the occasion, would suggest the capacity of pluripotent stem cells to differentiate along alternative pathways depending on local cues provided by different tissue environments, or in response to specific recruitment signals. Do these discoveries provide the basis of an entirely new concept of cell therapy, based on transplantation of adult pluripotent stem cells that can be induced to differentiate into specific tissues? A number of issues need to be addressed to answer this crucial question.
First, do pluripotent stem cells play a real role in adult tissue homeostasis, or are they just a remnant of embryonic and fetal life that is rapidly lost after birth? Definitive hematopoietic stem cells derive from an earlier embryonic precursor with both angiogenic and hematopoietic potential, which develops first in the embryonic aorta-gonad-mesonephros (AGM) region and then migrates into the fetal liver, the spleen, and eventually the bone marrow (reviewed in ref. 31). We found, to our surprise, that cells with the typical morphology of mouse adult satellite cells derive largely from the embryonic dorsal aorta and not from the somites, the presumed source of all skeletal myogenic cells (32). In vitro, these aorta-derived myogenic cells express a number of myogenic and endothelial markers that are also expressed by satellite cells, including a receptor for vascular endothelial growth factor (VEGF). VEGF receptor is also expressed on a fraction of murine (where it is named flk-1) and human (KDR) hematopoietic stem cells. In vivo, aorta-derived myogenic progenitors participate in muscle regeneration and fuse with resident satellite cells (32). These data suggest that a subset of postnatal myogenic cells may be rooted in a vascular lineage. Whether these myogenic/vascular cells arise from a primordial pericyte; from endothelial cells proper, as suggested by the expression of endothelial markers; or from a circulating endothelial progenitor (33) is not currently known (Figure 1). Also unknown is the lineage relationship, if any, of these cells with the primitive hemangioblast. The existence of a self-renewing, pluripotent hemato-angio-myogenic stem cell throughout embryonic, fetal, and at least early postnatal life is suggested, on one hand, by the presence of myogenic progenitors in all hematopoietic tissues, i.e., dorsal aorta, AGM, fetal liver, and bone marrow (ref. 32, and our unpublished observations), and on the other hand by

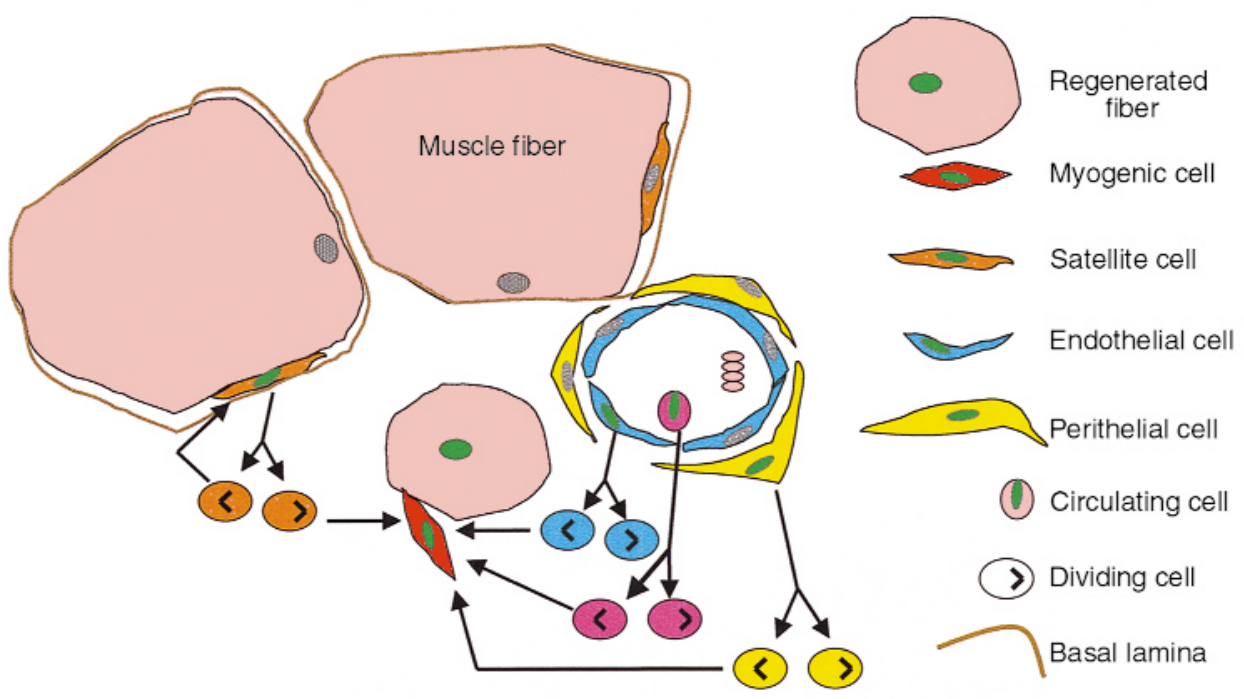

Figure 1

A schematic representation of the possible origin of myogenic cells in regenerating skeletal muscle. Regenerated fibers (characterized by centrally located nuclei) are formed by fusion of myogenic cells (dotted red) that may derive from resident, activated satellite cells (orange) or from cells derived from the microvasculature, either as circulating cells (violet), or as endothelial cells (light blue) or pericytes (yellow) of the vessel wall. A green nucleus symbolizes the activation of a myogenic program. 
the recovery of hematopoietic stem cells from heavily vascularized tissues, such as muscle $(27,28)$ or brain $(30)$. Analysis of mesodermal markers such as brachyury and flk-1 in differentiating ES cells indeed suggests the existence of a hierarchy of progenitors stemming from a primitive, possibly pluripotent mesodermal stem cell (34). Pluripotent stem cells may have myogenic or even additional potentials, since clones of dorsal aorta cells can give rise to osteoblast-like cells in the presence of $B M P 2$, a property retained by adult satellite cells (35). It is tempting to speculate that, during embryogenesis, pluripotent stem cells might colonize developing tissues through the vasculature and adopt specific fates, becoming satellite cells in the muscle or hematopoietic and stromal stem cells in the bone marrow, depending on local environmental cues. If such "plastic" stem cells are preserved in the microvasculature of adult tissues, as the recent transplantation experiments would suggest, will we ever be able to identify, to expand, and to transplant them in reasonable numbers? Analysis of the surface markers so far has not provided evidence for a unique phenotype for pluripotent stem cells. Although specific surface molecules (CD45, CD34, flk-1, Sca-1) have been identified, they vary depending on the tissue of origin or the activation state and are not necessarily correlated with the presence or absence of pluripotentiality or other stem cell characteristics. The absence of unique markers and the impossibility of carrying out clonal analysis in the only available assay of pluripotentiality, transplantation in vivo, clearly limit our ability to study the plasticity of these pluripotent stem cells.

Second, can we induce expansion and differentiation of pluripotent stem cells into a desired progeny in vivo with reasonable efficiency, using any of the diverse growth factors, cytokines, and chemokines now available? Cytokine and chemokine effects on the recruitment and differentiation of transplanted pluripotent cells are just beginning to be investigated, but insights from this work will clearly be important for clinical purposes, since it is unlikely that we will be able to recreate for nonhematopoietic tissues the selective ablation and competitive repopulation conditions that allowed bone marrow transplantation to become a clinical reality in the treatment of hematopoietic disorders.

Third, do pluripotent stem cells exist in humans, and how can we assay for their abundance, plasticity, and clinical potential? These are extremely difficult questions to answer, since in vitro assays to date have been unsatisfactory even for hematopoietic stem cells. Furthermore, direct injection into immunodeficient mice might allow testing of the muscle-forming capacity of human cells isolated from the bone marrow, but not of their selfrenewing capacity, plasticity, and recruitment through the peripheral circulation. Immunodeficient mouse strains that allow maintenance and expansion of very early human blood progenitors are currently available (e.g., the NOD/scid mouse) but do not support complete differentiation of all the hematopoietic progenies and are therefore unlikely to allow the analysis of even more complex phenomena such as recruitment and differentiation of a bone marrow-derived myogenic progenitor. More sophisticated human/mouse chimera are needed for this purpose, while other preclinical models, such as nonhuman primates, need to be tested to model more closely the human situation. Unfortunately, satisfactory models of human muscular dystrophy do not currently exist, and the dystrophic dog model is very expensive, is not easily available, and lacks specific immunological and molecular tools. We could therefore face the possibility of having to start clinical experiments directly in humans, first to address limited and specific biological questions, and eventually to assay for the muscle-replacing capacity of bone marrow cells in a relevant clinical background. Given the current primitive knowledge of the biology of marrow-derived myogenic progenitors, it is clearly premature to think about human clinical applications. Nevertheless, in the meantime, a consensus as to what knowledge we can gain from murine or any other model should be reached, and a consistent ethical framework to carry out future human trials should be developed as we begin to move in that direction.

\section{What do we expect in the future?}

Selection of a stem cell population with the required characteristics of pluripotentiality appears to be an obvious step toward developing bone marrow transplantation as a therapy for primary myopathies. As discussed above, classical bone marrow transplantation is monitored by the reconstitution of the hematopoietic compartment, but this is not informative for the reconstitution of a pluripotent stem cell or of a myogenic progenitor pool. Indeed, if the same stem cell is responsible for the reconstitution of the hematopoietic and the myogenic capacities, then the chances that transplantation conditions may be improved by enriching for more primitive cells become slim. Instead, we may be better advised to look for factors or conditions that allow selective expansion of the myogenic progeny in vivo, a condition that, for the hematopoietic compartment, is usually generated by radiation and myeloablation. If the myogenic stem/progenitor cell is distinct, or separable, from the hematopoietic stem cell, then it should be possible to increase its abundance in the bone marrow transplant by a number of measures, although it will still be necessary to find the right conditions for expansion and recruitment in vivo.

The most attractive scenario for clinical application requires an abundant source of autologous stem cells, which could be corrected genetically by inserting a functional, appropriately regulated copy of a missing gene (dystrophin, for instance) and then transplanted in sufficient numbers by an appropriate delivery route. Ideally, both myogenic differentiation and self-renewing capacity should be maintained in vivo. We are far from this ideal situation even for the hematopoietic stem cell, let alone for the still-elusive pluripotent or myogenic entity. Allogeneic bone marrow transplantation may provide a reasonable alternative, once we are in a position to predict significant muscle replacement capacity. The treatments currently associated with clinical bone marrow transplantation involve radiation and the use of toxic myeloablative drugs and imply risks that must seriously be considered for noncancer patients. Nevertheless, without the requirement for cancer cell ablation, milder protocols might be acceptable for fatal diseases like DMD. Allo- 
geneic bone marrow transplantation from a genetically normal donor could provide an additional but crucial benefit to dystrophic patients, tolerizing them to exogenous dystrophin and reducing the risk of immune rejection of dystrophin-producing cells.

Stem cell biology is clearly coming of age, and it is raising new hopes that a better fundamental knowledge, along with appropriate translational research, might finally provide the tools for the treatment of muscular dystrophy with cell and gene therapy. We will not need to wait too long to learn whether this is just wishful thinking or an entirely new therapeutic strategy.

\section{Acknowledgments}

Work in the authors' laboratories has been supported by the Italian Telethon, the European Community, and the Fondazione Cenci-Bolognetti in Rome, Italy.

1. Nawrotzki, R., Blake, D.J., and Davies, K.E. 1996. The genetic basis of neuromuscular disorders. Trends Genet. 12:294-298.

2. Miller, J.B., Schaefer, L., and Dominov, J.A. 1999. Seeking muscle stem cells. Curr. Top. Dev. Biol. 43:191-219.

3. Kochanek, S., et al. 1996. A new adenoviral vector: replacement of all viral coding sequences with $28 \mathrm{~kb}$ of DNA independently expressing both full-length dystrophin and beta-galactosidase. Proc. Natl. Acad. Sci. USA. 93:5731-5736.

4. Tinsley, J.M., et al. 1996. Amelioration of the dystrophic phenotype of $\mathrm{mdx}$ mice using a truncated utrophin transgene. Nature. 384:349-353.

5. Partridge, T.A., Beauchamp, J.R., and Morgan, J.E. 1989. Conversion of $m d x$ myofibers from dystrophin-negative to -positive by injection of normal myoblasts. Nature. 337:176-179.

6. Beauchamp, J.R., Pagel, C.N., and Partridge, T.A. 1997. A dual-marker system for quantitative studies of myoblast transplantation in the mouse. Transplantation. 63:1794-1797.

7. Ohtsuka, Y., Udaka, K., Yamashiro, Y., Yagita, H., and Okumura, K. 1998. Dystrophin acts as a transplantation rejection antigen in dystrophin-deficient mice: implication for gene therapy. J. Immunol. 160:4635-4640.

8. Qu, Z., et al. 1998. Development of approaches to improve cell survival in myoblast transfer therapy. J. Cell Biol. 142:1257-1267.

9. Beauchamp, J.R., Morgan, J.E., Pagel, C.N., and Partridge, T.A. 1999. Dynamics of myoblast transplantation reveal a discrete minority of precursors with stem cell-like properties as the myogenic source. $J$. Cell Biol. 144:1113-1122.

10. Guerette, G., et al. 1997. Prevention by anti-LFA-1 of acute myoblast death following transplantation. J. Immunol. 159:2522-2531.

11. Kinoshita, I., et al. 1996. Myoblast transplantation in monkeys: control of immune response by FK-506. J. Neuropathol. Exp. Neurol. 55:687-697.

12. Blau, H.M., and Springer, M.L. 1997. Muscle-mediated gene therapy. N. Engl. J. Med. 333:1554-1556.

13. Decary, S., et al. 1997. Replicative potential and telomere length in human skeletal muscle: implication for myogenic cell-mediated gene therapy. Hum. Gene Ther. 4:713-723.

14. Salvatori, G., et al. 1993. Retroviral vector-mediated gene transfer into human primary myogenic cells leads to expression in muscle fibers in vivo. Hum. Gene Ther. 4:713-723.
15. Huard, J., Verreault, S., Roy, R., Tremblay, M., and Tremblay, J.P. 1994. High efficiency of muscle regeneration after human myoblast clone transplantation in SCID mice. J. Clin. Invest. 93:586-599.

16. Simon, L.V., Beauchamp, J.R., O'Hare, M., and Olsen, I. 1996. Establishment of long-term myogenic cultures from patients with Duchenne muscular dystrophy by retroviral transduction of a temperature-sensitive SV40 large T antigen. Exp. Cell Res. 224:264-271.

17. Salvatori, G., et al. 1997. A temperature conditional mutant of SV40 large $T$ antigen requires serum to inhibit myogenesis and does not induce DNA synthesis in myotubes. Cell Growth Differ. 8:157-164.

18. Berghella, L., et al. 1999. Reversible immortalization of human myogenic cells by site-specific excision of a retrovirally transferred oncogene. Hum. Gene Ther. 10:1607-1617.

19. Lattanzi, L., et al. 1998. High efficiency myogenic conversion of human fibroblasts by adenoviral vector-mediated MyoD gene transfer. An alternative strategy for ex vivo gene therapy of primary myopathies. J. Clin. Invest. 101:2119-2128.

20. Huard, C., et al. 1998. Transplantation of dermal fibroblasts expressing MyoD1 in mouse muscles. Biochem. Biophys. Res. Commun. 248:648-654.

21. Cossu, G. 1997. Unorthodox myogenesis: possible developmental significance and implications for tissue histogenesis and regeneration. Histol. Histopathol. 12:755-760.

22. Kelly, R., Alonso, S., Tajbakhsh, S., Cossu, G., and Buckingham, M. 1995. Myosin light chain 3F regulatory sequences confer regionalized cardiac and skeletal muscle expression in transgenic mice. J. Cell Biol. 129:383-396.

23. Prockop, D.J. 1997. Marrow stromal cells as stem cells for nonhematopoietic tissues. Science. 276:71-74.

24. Bianco, P., and Robey, P.G. 2000. Marrow stromal stem cells. J. Clin. Invest. 105:1663-1668.

25. Bianco, P., and Cossu, G. 1999. Uno, nessuno e centomila: searching for the identity of mesodermal progenitors. Exp. Cell. Res. 251:257-263.

26. Ferrari, G., et al. 1998. Muscle regeneration by bone marrow-derived myogenic progenitors. Science. 279:1528-1530.

27. Gussoni, E., et al. 1999. Dystrophin expression in the $\mathrm{mdx}$ mouse restored by stem cell transplantation. Nature. 401:390-394.

28. Goodell, M.A., et al. 1997. Dye efflux studies suggest that hematopoietic stem cells expressing low or undetectable levels of CD34 antigen exist in multiple species. Nat. Med. 3:1337-1345

29. Jackson, K.A., Mi, T., and Goodell, M.A. 1999. Hematopoietic potential of stem cells isolated from murine skeletal muscle. Proc. Natl. Acad. Sci. USA. 96:14482-14486.

30. Bjornson, C.R., Rietze, R.L., Reynolds, B.A., Magli, M.C., and Vescovi, A.L. 1999. Turning brain into blood: a hematopoietic fate adopted by adult neural stem cells in vivo. Science. 283:534-537.

31. Dzierzak, E., Medvinsky, A., and de Bruijn, M. 1998. Qualitative and quantitative aspects of haematopoietic cell development in the mammalian embryo. Immunol. Today. 19:228-236.

32. De Angelis, L., et al. 1999. Skeletal myogenic progenitors originating from embryonic dorsal aorta coexpress endothelial and myogenic markers and contribute to postnatal muscle growth and regeneration. J. Cell Biol. 147:869-878.

33. Asahara, T., et al. 1999. Bone marrow origin of endothelial progenitor cells responsible for post-natal vasculogenesis in physiological and pathological neovascularization. Circ. Res. 85:221-228.

34. Robertson, S.M., Kennedy, M., Shannon, J.M., and Keller, G. 2000. A transitional stage in the commitment of mesoderm to hematopoiesis requiring the transcription factor SCL/tal-1. Development. 127:2447-2459.

35. Katagiri, T., et al. 1994. Bone morphogenetic protein- 2 converts the differentiation pathway of $\mathrm{C} 2 \mathrm{C} 12$ myoblasts into the osteoblast lineage. J. Cell Biol. 127:1755-1766. 